\title{
Risk factors for infection and the effect of an institutional shunt protocol on the incidence of ventricular access device infections in preterm infants
}

\author{
Heather S. Spader, MD, ${ }^{1}$ Dean A. Hertzler, MD, ${ }^{2}$ John R. W. Kestle, MD, ${ }^{3}$ \\ and Jay Riva-Cambrin, MD, MSc ${ }^{3}$

\begin{abstract}
1'Department of Neurosurgery, Rhode Island Hospital, Brown University, Providence, Rhode Island; '2Department of Neurosurgery, Joe DiMaggio Children's Hospital, Hollywood, Florida; and 'Department of Neurosurgery, Division of Pediatric Neurosurgery, Primary Children's Hospital, University of Utah, Salt Lake City, Utah
\end{abstract}

\begin{abstract}
OBJECT Intraventricular hemorrhage in premature infants often leads to progressive ventricular dilation and the need for ventricular reservoir placement. Unfortunately, these reservoirs have a higher rate of infection than ventriculoperitoneal shunts in premature babies. The authors analyzed the risk factors for infection in this population and studied whether the implementation of an institutional protocol for shunt placement had a corollary effect on ventricular access device (VAD) infection rates in premature neonates with intraventricular hemorrhage.

METHODS The authors conducted a retrospective cohort review of consecutive premature neonates in whom VADs were inserted in the operating room at Primary Children's Hospital between June 2003 and June 2011 to identify risk factors for infection. Medical records were reviewed for information on infection (culture proven or eroded hardware at 90 days), gestational age at birth, weight, gestational age at surgery, intrathecal antibiotics, hemorrhage, death, and surgeon. The institution used a pilot protocol for shunt infection reduction in 2006-2007, and then the full Hydrocephalus Clinical Research Network protocol from June 2007 to 2011, and the rates of infection during these periods were analyzed. Confounding factors such as sepsis, necrotizing enterocolitis, and a history of meningitis were also analyzed.

RESULTS The overall infection rate was 10.5\% (11 patients) in the 105 patients identified. Gestational age at procedure was a significant risk factor for infection $(p=0.05)$. Meningitis was significantly associated with infection, with $63 \%$ of the infected group having had prior meningitis compared with $7 \%$ for the noninfected group $(p<0.001)$. Concurrent with the implementation of the protocol to reduce shunt infection, the VAD infection rate decreased from $14.7 \%$ to $5.4 \%(p=0.2)$. CONCLUSIONS Gestational age at procedure and previous meningitis were significant risk factors for VAD infections. In addition, the implementation of an institutional standardized shunt protocol for ventriculoperitoneal shunts may have altered the operating room team's behavior, indicated by a nonmandated use of intrathecal antibiotics in VAD surgeries, contributing to a reduced VAD infection rate. Although the observed difference was not statistically significant with the small sample size, the authors believe that these findings deserve further study.
\end{abstract}

http://thejns.org/doi/abs/10.3171/2014.9.PEDS14215

KEY WORDS infection; hydrocephalus; ventricular hemorrhage; ventricular access device

I NTRAVENTRICULAR hemorrhage (IVH) from germinal matrix hemorrhage is a frequent and problematic consequence of premature delivery. Historic rates of hemorrhage have ranged from $15 \%$ to $39 \%$ of preterm births in neonates weighing less than $1.5 \mathrm{~kg} .^{2}$ Advances in neonatal care in the last decade have reduced this rate to $15 \%-20 \% .^{3,8}$ Neonates with IVH suffer from neurological insults, one of the most life-threatening of which is pro- gressive posthemorrhagic ventricular dilation (PPHVD), which affects $25 \%-50 \%$ of infants with IVH. ${ }^{8}$ This complication usually occurs within the first 10-20 days after initial hemorrhage and is manifested clinically by increasing head circumference, diastasis of cranial sutures, and full, bulging fontanelles. This dilation often requires neurosurgical intervention with the placement of a ventricular access device (VAD) to allow serial drainage of CSF to

ABBREVIATIONS HCRN = Hydrocephalus Clinical Research Network; IT = intrathecal; IVH = intraventricular hemorrhage; NEC = necrotizing enterocolitis; PCH = Primary Children's Hospital; PPHVD = progressive posthemorrhagic ventricular dilation; VAD = ventricular access device; VP = ventriculoperitoneal.

SUBMITTED May 2, 2014. ACCEPTED September 4, 2014.

INCLUDE WHEN CITING Published online December 5, 2014; DOI: 10.3171/2014.9.PEDS14215.

DISCLOSURE The authors report no conflict of interest concerning the materials or methods used in this study or the findings specified in this paper. 
control head growth and to treat the hydrocephalus. The VAD is used until the premature infant demonstrates a need for a permanent ventriculoperitoneal (VP) shunt and is large enough to tolerate the placement procedure.

Infections of VADs are common, and the prevalence has been reported in the literature at rates from approximately 5\%-39\%, with most studies reporting an approximate $5 \%-15 \%$ rate of infection., ${ }^{1,2,79}$ Infections of VADs can delay permanent shunt implantation and decrease the functional outcome in these infants. One study that examined preoperative antibiotic use found that VAD infection rates decreased $20 \%$ with the introduction of preoperative antibiotics. ${ }^{2}$ Another study looking at serial taps of VADs found that additional taps do not necessarily correlate with increased infection rates. ${ }^{5} \mathrm{~A}$ recent study by Kestle et al. ${ }^{4}$ showed that the institution of a protocol for placement of VP shunts decreased infection rates from $8.8 \%$ to $5.7 \%$ $(p=0.0028)$ across multiple institutions. In this study, we hypothesized that the implementation of a shunt insertion protocol at our institution in 2007 would have a parallel effect of decreasing infections with other ventricular implants such as VADs as a result of institutional culture change.

\section{Methods \\ Study Design}

This was a single-center retrospective cohort study undertaken with the approval of the Primary Children's Hospital (PCH) Privacy Board and the University of Utah Institutional Review Board, with a waiver of consent. All data were obtained from $\mathrm{PCH}$ paper and electronic charts. Ten percent of charts were reviewed by 2 reviewers to ensure the accuracy of data abstraction.

\section{Study Population}

Neonates in whom VADs were placed in the operating room during the period from June 1, 2003, to June 1, 2011, were identified. All VADs were preconnected by the manufacturer and none were impregnated with antibiotics. The cohort was assembled by interrogating the pediatric neurosurgical case database and cross-referencing with a billing database to ensure a consecutive sample of infants who underwent the insertion of a VAD at our institution. Preterm (less than 37 weeks gestation) infants with Grade III or IV IVH and PPHVD on imaging who underwent the implantation of a VAD, otherwise known as a ventricular reservoir, were included. Infants were excluded if they were temporized with a subgaleal shunt or died within 1 month of birth.

\section{Outcome Variables}

The primary outcome variable was infection of the VAD within 90 days of placement. All patient records were reviewed for 1 year after insertion to ensure detection of any delayed infections or complications. Infection was defined as a positive CSF culture sampled from the implant, a positive open implant wound culture, or exposed hardware. In all cases of confirmed infection, the VAD was surgically removed and serial lumbar punctures were used to control PPHVD until the infection was clinically cleared.
Additional variables in the analysis were gestational age (in weeks), birth weight (in grams), weight at procedure (in grams), gestational age (in weeks) at VAD insertion, postoperative hemorrhage as seen on imaging, death, surgeon, and 90-day infection outcome. In addition, patients with documented sepsis at any time during their hospitalizations, medical and surgical necrotizing enterocolitis (NEC) as documented by the neonatologists, and meningitis treated with antibiotics were also noted. All patients who had meningitis received a full course of antibiotics and had a negative CSF culture prior to VAD insertion.

Infections were also analyzed as they related to the implementation of a shunt insertion protocol at $\mathrm{PCH}$. Prior to 2006, PCH had no specific infection control protocol for shunt surgery or VAD insertion. In 2006, the pediatric neurosurgeons self-mandated a pilot protocol that included the use of intrathecal (IT) antibiotics at the conclusion of all shunt surgeries, although not necessarily VAD insertions. A more extensive protocol was adopted by the Hydrocephalus Clinical Research Network (HCRN) on June 1, 2007. The HCRN protocol similarly included IT antibiotics as well as other infection prevention behaviors. Although neither shunt insertion protocol was required for VAD surgery, we hypothesized that the surgical and operating room teams might have changed their behavior as a result of their shunt infection protocol experience and applied some of the same methods when doing VAD surgery. Because IT antibiotics were not used during VAD surgery prior to adoption of either protocol, we recorded their use during VAD surgery as a marker of change in behavior. The use of IT antibiotics was obtained from the anesthesia record. In analyzing the effect of a shunt insertion protocol on VAD infections, the data were divided into 3 eras: preprotocol (before 2006), $\mathrm{PCH}$ pilot protocol (2006-2007), and post-HCRN protocol adoption (beginning in June 2007).

\section{Statistical Methods}

Univariate results were expressed as counts and percentages for categorical variables and as means and SDs for continuous variables. The chi-square and Fisher exact tests were used for bivariate analyses for categorical variables, whereas t-tests were used for bivariate analyses for the continuous variables. The $p$ values were reported for all of these analyses. Multivariate logistic regression was constructed to predict VAD infection by using significant bivariate factors as well as the variable of interest (era of infection protocol). Results from the final model were reported as $\mathrm{p}$ values and adjusted odds ratios with $95 \%$ confidence intervals. Statistical analyses were conducted using SAS (version 9.2).

\section{Results}

A total of 105 patients were identified who met our criteria: 34 patients before 2006, 34 during 2006-2007, and 37 after June 1, 2007. Their average gestational age at birth (mean $\pm \mathrm{SD}$ ) was $27.9 \pm 3.3$ weeks, the mean gestational age at procedure was $31.9 \pm 16.2$ weeks, the mean birth weight was $1195 \pm 497 \mathrm{~g}$, and the mean weight at procedure was $1600 \pm 559 \mathrm{~g} ; 65 \%$ were male (Table 1). A total 
of 12 infants had NEC (11.4\%), of whom 7 (58\%) required abdominal surgery. In our overall sample, $49 \%$ were treated for presumed sepsis during hospitalization, and $13.5 \%$ had a history of meningitis. There were 3 deaths and no new hemorrhages after placement. The use of IT antibiotics started at $0 \%$, was $87.5 \%$ by 2007 , and was $100 \%$ by 2009 (Fig. 1). This correlates with our time frame of preprotocol, implementation of protocol, and post-HCRN protocol (Table 2).

The overall infection rate was 11 of 105 (10.5\%). Gestational age at procedure was a significant risk factor for VAD infection, with the average age of 26.4 weeks in the infected cohort compared with a mean age of 32.6 weeks for the noninfected group $(p=0.05)$. Meningitis was a significant risk factor for infection; $63 \%$ of the infected group had prior meningitis, whereas only $7 \%$ of the uninfected group had meningitis previously $(\mathrm{p}<0.001)$. Meningitis was also significantly associated with death $(\mathrm{p}=0.006)$. All the other variables were not significantly associated with VAD infection (Table 3).

Infection rates by protocol era were as follows: $14.7 \%$ preprotocol, $11.8 \%$ during implementation, and $5.4 \%$ postHCRN shunt infection protocol $(\mathrm{p}=0.2)$. The absolute risk reduction was $9.3 \%$ in the preprotocol group compared with the postprotocol group, and the relative risk reduction was $63.3 \%$ (Table 4 ).

When gestational age at procedure, a prior history of meningitis, and protocol era were analyzed using multivariate logistic regression, only a history of meningitis was independently associated with VAD infection (OR 21.5, 95\% CI 5.0-91.7).

\section{Discussion}

We present a retrospective cohort study of 105 neonates in whom VADs were placed for PPHVD. Eleven of these

TABLE 1. Description of overall cohort of 105 patients with VADs*

\begin{tabular}{lc}
\hline \multicolumn{1}{c}{ Demographic \& Clinical Variables } & Study Cohort \\
\hline Age at procedure, in wks (mean \pm SD) & $31.9 \pm 16.2$ \\
\hline Gestational age in wks (mean \pm SD) & $27.9 \pm 3.3$ \\
\hline Male sex & $68(65)$ \\
\hline Birth weight in g (median \pm IQR) & $1195 \pm 497$ \\
\hline Weight at procedure, in g (median \pm IQR) & $1600 \pm 559$ \\
\hline Surgeon & $26(24.8)$ \\
\hline 1 & $29(27.6)$ \\
\hline 2 & $33(31.4)$ \\
\hline 3 & $16(15.2)$ \\
\hline 4 & $1(1)$ \\
\hline 5 & $12(11.4)$ \\
\hline NEC & $51(49)$ \\
\hline Presumed sepsis & $14(13.5)$ \\
\hline Meningitis & $0(0)$ \\
\hline Postprocedural hemorrhage & $3(2.9)$ \\
\hline Death & $11(10.5)$ \\
\hline VAD infection & \\
\hline IQR = interquartile range. & \\
$*$ & Values are expressed as the number (\%) unless otherwise specified.
\end{tabular}

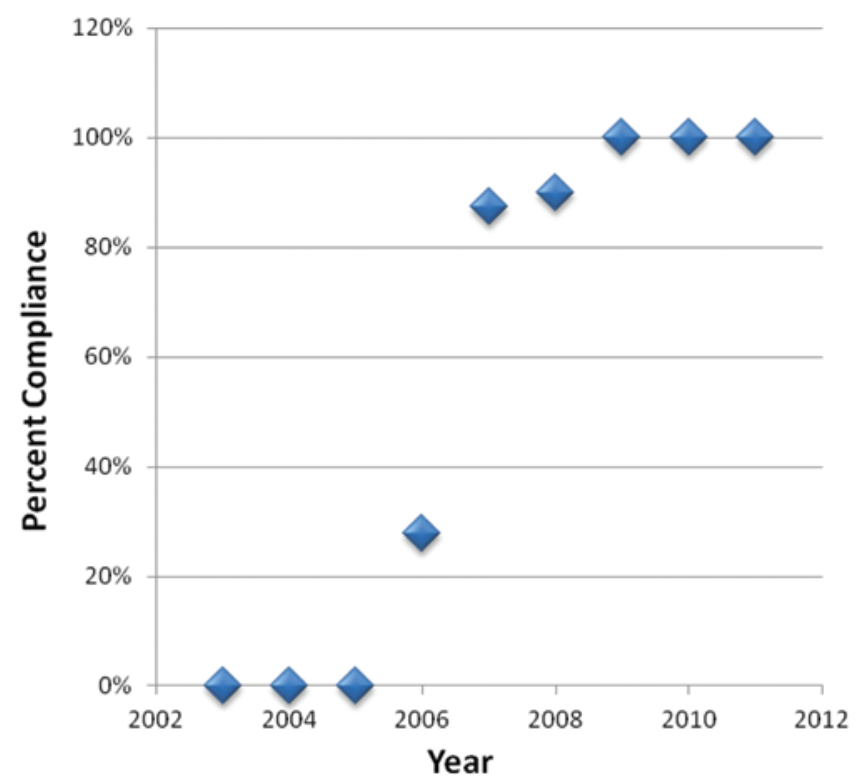

FIG. 1. Graph showing use of antibiotics as a marker for compliance with the HCRN protocol. Figure is available in color online only.

patients subsequently experienced a VAD infection, for an overall infection rate of $10.5 \%$. Bivariate analyses showed that gestational age at procedure $(\mathrm{p}=0.05)$ and meningitis $(p<0.001)$ were significant risk factors for VAD infection. In addition, meningitis was also significantly associated with death $(\mathrm{p}=0.006)$.

Two previous studies have examined risk factors for infection in VADs and VP shunts in infants. The first, by Pople et al. ${ }^{6}$ in 1992, found a VP shunt infection rate of $15.7 \%$ for infants less than 6 months old. This study found that age was the only major factor influencing the infection rate. A more recent study by Bruinsma et al. ${ }^{2}$ found an infection rate of $33.3 \%$ in preterm infants with VADs or VP shunts. Of note, this study enrolled 39 preterm infants and is the largest study of infections associated with preterm infants with VADs/VP shunts in the literature. The authors concluded that prematurity and extremely low birth weight were significant factors contributing to VAD/ VP shunt infections.

TABLE 2. Annual antibiotic compliance with the shunt and infection protocol

\begin{tabular}{cccl}
\hline $\begin{array}{c}\text { Yr VAD } \\
\text { Placed }\end{array}$ & $\begin{array}{c}\text { No. of VADs } \\
\text { Placed }\end{array}$ & $\begin{array}{c}\text { IT Antibiotic } \\
\text { Compliance (\%) }\end{array}$ & Protocol Era \\
\hline 2003 & 6 & 0 & Preprotocol \\
\hline 2004 & 10 & 0 & Preprotocol \\
\hline 2005 & 18 & 0 & Preprotocol \\
\hline 2006 & 18 & 27.8 & PCH pilot protocol \\
\hline 2007 & 16 & 87.5 & $\begin{array}{c}\text { PCH pilot protocol/post- } \\
\text { HCRN protocol }\end{array}$ \\
\hline 2008 & 10 & 90 & Post-HCRN protocol \\
\hline 2009 & 12 & 100 & Post-HCRN protocol \\
\hline 2010 & 13 & 100 & Post-HCRN protocol \\
\hline 2011 & 2 & 100 & Post-HCRN protocol \\
\hline
\end{tabular}


TABLE 3. Univariate analysis of infection risk factors in 105 patients with VADs*

\begin{tabular}{lccc}
\hline \multicolumn{1}{c}{ Demographic \& Clinical Variables } & Patients w/o Infection & Patients w/ Infection & p Value \\
\hline No. of patients & 94 & 11 & 0.05 \\
\hline Gestational age at procedure, in wks (mean \pm SD) & $32.6 \pm 16.8$ & $26.4 \pm 8.2$ & NS \\
\hline Male sex & $63(67)$ & $5(45.5)$ & NS \\
\hline Weight at procedure, in g (mean \pm SD) & $1609 \pm 580$ & $1521 \pm 341$ & NS \\
\hline Gestational age at birth, in wks (mean \pm SD) & $27.9 \pm 3.4$ & $27.9 \pm 1.9$ & NS \\
\hline Birth weight in g (mean \pm SD) & $1200 \pm 520$ & $1146 \pm 240$ & NS \\
\hline NEC & $11(11.7)$ & $1(9.1)$ & NS \\
\hline Sepsis & $43(45.7)$ & $8(72.7)$ & NS \\
\hline Meningitis & $7(7.5)$ & $7(63.6)$ & NS \\
\hline IT antibiotics & $49(52.1)$ & $6(54.6)$ & 0.2 \\
\hline Death & $2(2.1)$ & $1(9.1)$ & \\
\hline Era & & $5(45.5)$ & $4(36.4)$ \\
\hline Preprotocol & $29(30.9)$ & $2(18.2)$ & NS \\
\hline PCH pilot protocol & $30(31.9)$ & & NS \\
\hline Post-HCRN protocol & $35(37.2)$ & $2(18.2)$ & NS \\
\hline Surgeon & & $3(27.3)$ & NS \\
\hline 1 & $24(25.5)$ & $3(27.3)$ & $2(18.2)$ \\
\hline 2 & $26(27.7)$ & $1(9.1)$ & \\
\hline * & $30(31.9)$ & & \\
\hline 4 & $14(14.9)$ & & \\
\hline Values are expressed as the number (\%) unless otherwise specified. & & & \\
\hline
\end{tabular}

\section{Age as Risk Factor}

Our study of 105 neonates is the largest series studying infection risk factors in preterm infants requiring neurosurgical intervention for CSF diversion. Like previous investigators, we found that age at the time of implantation was a risk factor for infection, with a significantly increased risk of infection in younger neonates (26 weeks vs 33 weeks, $\mathrm{p}=0.05){ }^{2,6}$ We expected that younger age was significant because of the higher risk of comorbidities such as NEC and sepsis that comes with prematurity; however, our analyses demonstrated that these other risk factors were not significantly associated with VAD infection. Younger neonates are probably predisposed to infection because of a combination of a more fragile immune system and thinner skin overlying the protuberant hardware.

\section{Meningitis as Risk Factor}

A history of meningitis was also significantly associated with infection $(\mathrm{p}<0.001)$ in both the bivariate and

TABLE 4. Infection rate in 105 patients with VADs, by protocol era

\begin{tabular}{lccc}
\hline \multicolumn{1}{c}{ Variable } & $\begin{array}{c}\text { Preprotocol } \\
\text { (pre-2006) }\end{array}$ & $\begin{array}{c}\text { PCH Pilot } \\
\text { Protocol } \\
(2006-2007)\end{array}$ & $\begin{array}{c}\text { Post-HCRN } \\
\text { Protocol (June }\end{array}$ \\
2007-present)
\end{tabular}

the multivariate analyses. It is interesting to note that meningitis was associated with infection, whereas NEC or presumed sepsis was not, because these conditions would also seem to be significant risk factors for VAD infections. The lack of other infection-related variables may be due to the relatively small sample size of infected infants; however, we did find that meningitis is a clear risk factor and that it may be worthwhile to defer VAD placement as long as possible in patients with meningitis. Conversely, it may be safer than previously thought to place VADs in infants with a recent history of NEC or other systemic infections.

\section{New Protocol}

While we were studying VAD infection, a standardized protocol was introduced to reduce shunt infection; therefore, we also considered whether our efforts to reduce shunt infection had any impact on VAD infection. In fact, we did observe fewer VAD infections during this time, although the difference was not significant (decrease from $14.7 \%$ to $5.4 \%, \mathrm{p}=0.2$ ).

We found it interesting that the VAD infection rate seemed to decrease coincidentally with the introduction of the shunt infection protocol. Because culture change is an important component of quality improvement, it is possible that members of the operating room team may have changed their behavior as a result of their experiences with the shunt insertion protocol, and perhaps this contributed to the decreased infection rate. The only change in behavior during VAD operations that we could reliably identify in the medical record was IT antibiotic use, which 
increased from $0 \%$ to $87.5 \%$ to $100 \%$. Although antibiotic use alone did not impact infection rates in shunt surgery, ${ }^{4}$ it does appear to indicate a change in behavior during VAD procedures. This leads us to believe that a standard protocol for shunt surgery highlighted the infection issue and emphasized infection prevention, and may have contributed to reduced infections.

\section{Limitations of the Study}

The main limitation of this study is that it is a retrospective cohort study and not a prospective trial. Another significant limitation is that there were only 11 infections (outcomes) in this cohort, decreasing the power of this study. In this retrospective review, we were not able to assess operating room team behaviors accurately, and therefore our suggestion that the team's behavior changed as a result of the infection protocol is only a hypothesis. Nevertheless, we believe that the trends seen in this study warrant further multicenter prospective studies.

\section{Conclusions}

Neonates with PPHVD are at high risk for infection. Our study found that gestational age (less than 26 weeks) and a recent history of meningitis were statistically significant risk factors for VAD infection. In addition, we found that the implementation of a shunt insertion protocol at the institution may have had a clinical effect on the reduction of infections in this other patient population because of a change in the operating room culture that led to greater attention to infection prevention. We recommend a further multiinstitutional study on the effect of a protocol to decrease VAD infections in the premature infant population.

\section{References}

1. Brockmeyer DL, Wright LC, Walker ML, Ward RM: Management of posthemorrhagic hydrocephalus in the low-birthweight preterm neonate. Pediatr Neurosci 15:302-308, 1989

2. Bruinsma N, Stobberingh EE, Herpers MJ, Vles JS, Weber BJ, Gavilanes DA: Subcutaneous ventricular catheter reservoir and ventriculoperitoneal drain-related infections in preterm infants and young children. Clin Microbiol Infect 6:202-206, 2000
3. Hintz SR, Poole WK, Wright LL, Fanaroff AA, Kendrick DE, Laptook AR, et al: Changes in mortality and morbidities among infants born at less than 25 weeks during the postsurfactant era. Arch Dis Child Fetal Neonatal Ed 90:F128F133, 2005

4. Kestle JR, Riva-Cambrin J, Wellons JC III, Kulkarni AV, Whitehead WE, Walker ML, et al: A standardized protocol to reduce cerebrospinal fluid shunt infection: the Hydrocephalus Clinical Research Network Quality Improvement Initiative. J Neurosurg Pediatr 8:22-29, 2011

5. Kormanik K, Praca J, Garton HJ, Sarkar S: Repeated tapping of ventricular reservoir in preterm infants with posthemorrhagic ventricular dilatation does not increase the risk of reservoir infection. J Perinatol 30:218-221, 2010

6. Pople IK, Bayston R, Hayward RD: Infection of cerebrospinal fluid shunts in infants: a study of etiological factors. J Neurosurg 77:29-36, 1992

7. Richard E, Cinalli G, Assis D, Pierre-Kahn A, Lacaze-Masmonteil T: Treatment of post-haemorrhage ventricular dilatation with an Ommaya's reservoir: management and outcome of 64 preterm infants. Childs Nerv Syst 17:334-340, 2001

8. Robinson S: Neonatal posthemorrhagic hydrocephalus from prematurity: pathophysiology and current treatment concepts. J Neurosurg Pediatr 9:242-258, 2012

9. Wellons JC III, Shannon CN, Kulkarni AV, Simon TD, RivaCambrin J, Whitehead WE, et al: A multicenter retrospective comparison of conversion from temporary to permanent cerebrospinal fluid diversion in very low birth weight infants with posthemorrhagic hydrocephalus. J Neurosurg Pediatr 4:50-55, 2009

\section{Author Contributions}

Conception and design: Riva-Cambrin. Acquisition of data: all authors. Analysis and interpretation of data: all authors. Drafting the article: Spader, Hertzler. Critically revising the article: all authors. Reviewed submitted version of manuscript: all authors. Approved the final version of the manuscript on behalf of all authors: Riva-Cambrin. Study supervision: Riva-Cambrin, Kestle.

\section{Correspondence}

Jay Riva-Cambrin, Department of Neurosurgery, Division of Pediatric Neurosurgery, Primary Children's Hospital, University of Utah, 100 Mario Capecchi Dr., Salt Lake City, UT 84113. email: jay.riva-cambrin@hsc.utah.edu. 